\title{
Convergence Adjustment Method Based on Approximate Power Flow and Voltage Stability
}

\author{
Nan $\mathrm{Hu}^{1,}{ }^{1,}$, Lin Qiao ${ }^{1}$, Chao Yang ${ }^{1}$, Jun $\mathrm{Qi}^{1,2}$, Shiyan $\mathrm{Hu}^{2,3}$ \\ ${ }^{1}$ State Grid Liaoning Electric Power Supply Co., LTD. Information \& Telecommunication Branch, \\ Shenyang, 110004, Liaoning, China \\ ${ }^{2}$ Shenyang University of Technology, 110870, Liaoning, China \\ ${ }^{3}$ University of Southampton \\ *Corresponding Author: $\mathrm{Nan} \mathrm{Hu}$
}

\begin{abstract}
:
Prone to problems flow calculation does not converge at the scheduled maximum power operating mode, the current operating personnel inability to visually observe the system state, convergence can only be adjusted by trial and error approach. Solving the problem of non convergence of power flow plays an important role in power system analysis. Based on the intermediate process of solving the Newton method, the concept of an approximate fashion, the relation between convergence and voltage stability is calculated by analyzing the trend of the voltage characteristics similar trend as the main basis of the judgment result in the trend does not converge, based on voltage sensitivity further, by improving the convergence of the method to boost the voltage level indirectly. Finally, after the New England 39-bus system to verify, by demonstrating the relevance and accuracy of the proposed method. When not solve the problem of the convergence trend is not impressive, it has a certain reference value for practical application. This study has a good application prospect in practical engineering application
\end{abstract}

Keywords: Convergence, Approximation flow, Sensitivity, Voltage stability.

\section{INTRODUCTION}

When arranging the operation mode, it is necessary to simulate the system state under the maximum operation mode. Because of the factors of large scale and high load level, the problem of nonconvergence of power flow calculation often occurs. Because operators can't observe the system state directly, they can't adjust the convergence through the power system knowledge they are good at. The existing manual power flow adjustment methods only stay at the level of trial and error with experience, so the adjustment effect is not ideal.

The existing research on the convergence of power flow calculation focuses on the analysis 
of three factors which affect the convergence of power flow calculation, model parameters and injection data.

In terms of solving methods, many methods for solving nonlinear equations have been applied to power flow calculation among which Newton method is the most widely used [1]. In view of the sensitivity of Newton method to initial value, references [2,3] proposed to use other initial value insensitive calculation methods for initial iteration, so as to provide better initial value for Newton method; the convergence theorem of power flow calculation is proposed in [4], which is used to analyze the convergence problem of power flow caused by improper selection of initial value of Newton method. In order to improve the accuracy of calculation model, Japanese scholar Iwamoto first proposed a rectangular coordinate Newton algorithm with second order terms which has good convergence reliability and convergence speed [5]. In addition, some improved algorithms such as optimal multiplier method [6-8], nonlinear programming method [9-11], Levenberg-Marquardt [12-14] are applied to power flow calculation, and the range of solving power flow equation is further expanded.

In terms of model parameters, the research on the transformation of different types of nodes is of typical significance. Reference [15] introduced the conversion logic between PQ node and PV node in detail and cited the control relationship between reactive power and voltage as the improvement of node type conversion. The index of reactive power shortage and searching the nodes with insufficient reactive power can solve the problem of non- convergence caused by reactive power imbalance [16].

In terms of injection data, the typical research on improving power flow convergence from the perspective of injection data mainly relies on sensitivity method and nonlinear programming method. In the sensitivity method, reference [17] proposed to select and adjust the generator output, terminal voltage, load power and other variables to improve the convergence. In reference [18], the primal dual interior point method is used to solve the minimum nodal power cut off considering the system inequality constraints. In reference [19], the power factor of the unresectable load is fixed and the solving dimension is reduced by using the load shedding ratio instead of the load shedding amount as the objective function.

In terms of data injection, the typical method is to improve the convergence of power flow by means of sensitivity method and nonlinear programming method. Reference [17] proposed to select and adjust the generator output, terminal voltage, load power and other variables to improve convergence through sensitivity calculation. In reference [18], the objective function is to minimize the node power cut, and the inequality constraints are considered, and the primal dual interior point method is used to solve the problem. In reference [19], the load shedding ratio is used instead of the load shedding amount as the objective function. Meanwhile, the power factor of the unresectable load is fixed and the solution dimension is reduced.

In recent years, with the help of the intermediate variables in the iterative process, the research on the convergence of power flow has been paid more and more attention. References [20] defined a method for identifying ill conditioned features based on voltage attenuation index, and 
established a planning model based on the ill conditioned characteristics. Reference [21] proposed an index to measure the error between intermediate variables and injected data to quantitatively evaluate the gap between initial power flow and intermediate power flow.

This paper clarifies the relationship between power flow convergence and voltage stability and proposes a concept of approximate power flow. The method of increasing the voltage level indirectly improves the convergence by using the voltage characteristics of the approximated power flow as the main basis for judging the failure of the power flow. Finally, the IEEE39-bus system is simulated to verify the pertinence and accuracy of the proposed method.

\section{THE RELATIONSHIP BETWEEN POWER FLOW CONVERGENCE AND SYSTEM VOLTAGE STABILITY}

Mathematically, the non-convergence of power flow calculation shows that the power equation cannot be solved accurately and it shows that some electrical parameters are extremely over-limit which leads to the limit state of the power system and cannot operate safely and stably in physics. The surge of electricity load and the transmission mode of high-voltage, long-distance and large-capacity transmission tend to cause voltage instability problems. According to the bifurcation principle, a small increase in load at this time may cause a sharp drop in voltage. From a mathematical point of view, the above situation often occurs at the case that Jacobian matrix is close to the singularity which leads to nonconvergence of calculation.

Taking the PV curve of power system as an example, when the system load is at a low level, the voltage stability is good, and the conventional iterative method can easily obtain the system power flow; with the increase of the load level, the system voltage stability gradually deteriorates. If the power flow calculation is carried out, a reasonable initial value or other auxiliary calculation strategies should be given; when the load level continues to increase, the voltage level is greatly reduced, and the conventional power flow algorithm can not meet the calculation requirements. In this case, the continuation method and other means are needed to obtain the solution; finally, when the operating point touches the saddle node bifurcation point of PV curve, the voltage collapses, and the power flow equation has no solution completely.

Taking PV curve of power system as an example (as shown in figure 1), the voltage stability is good and the system power flow can be easily obtained by conventional iterative solution when the level of low is low. As the load level increases, the system voltage stability deteriorates gradually. At this time, a reasonable initial value or other auxiliary calculation strategy is needed for power flow calculation. When the load level continues to increase, the voltage level decreases greatly and the conventional power flow algorithm can no longer meet the calculation requirements. Finally, when the operating point reaches the saddle bifurcation point of the PV curve, the voltage collapses and the tide breaks down which leads to flow equation has no solution at all. 


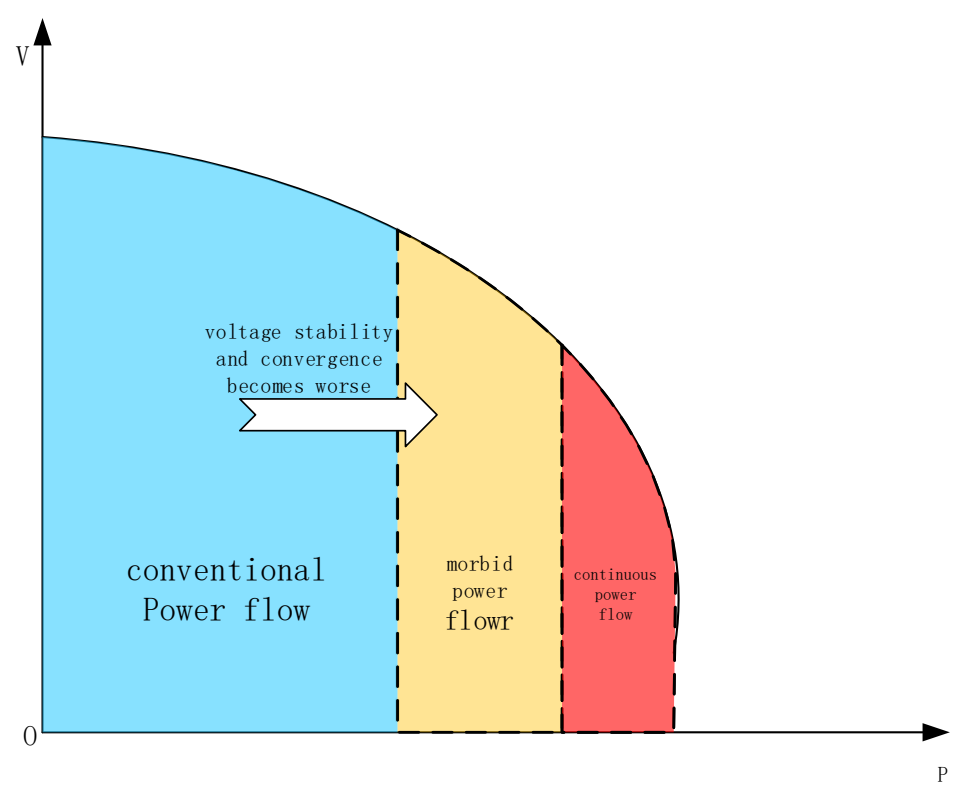

Fig 1: Schematic diagram of P-V curve

It can be found that the convergence of the power flow calculation and the voltage stability of the system can influence each other to some extent which can provide reference for the adjustment of the power flow convergence.

\section{ANALYSIS OF THE INTERMEDIATE PROCESS OF NEWTON METHOD ITERATION}

Newton iteration method is the most common computer solution for solving power flow equations in power systems. It produces a large number of intermediate variables in the calculation process, and the intermediate process of the current iteration contains important information.

Each iteration calculation is a process of linearizing the power flow equation. Therefore, a complete set of power flow forms can be obtained by perfecting the calculation of branches and balance nodes in each iteration which is called intermediate power flow in this article. Each electrical quantity in the intermediate power flow system satisfies the linearized power flow equation. It can be understood that under the condition of data injection that cannot be converged, if the convergence criterion is relaxed the iteration can approximately converge to a set of solutions with certain errors that is the intermediate power flow is a set of power flows that converge but have inadmissible errors in the injection space.

The difference between the intermediate power flow and the initial power flow is represented by $\Delta \mathrm{y}$. After $\Delta \mathrm{y}$ is given, $\Delta \mathrm{x}$ is solved through a modified equation and the initial value of the next iteration is updated with the help of $\Delta \mathrm{x}$ to establish the connection between the two iterations. In the process of solving, the change trend of the correction amount reflects the convergence 
ability of the iterative calculation. When the correction amount gradually tends to decrease until the accuracy requirements are met, the Newton iteration can achieve convergence.

\section{APPROXIMATE TIDAL CURRENT}

In order to obtain a power flow that is more fully close and convergent to the injected data, this paper proposes a concept of approximate power flow. Approximate power flow can provide reference for the convergence adjustment of the injected data by analogy with the physical problems that may exist in the similar power flow.

\subsection{Propose}

The approximate power flow is obtained by further narrowing the gap on the basis of the intermediate power flow. According to the iterative calculation principle, the injected power will be more or less gaps between the intermediate power flow and the intermediate power flow. Under extreme conditions, it is assumed that the injected data is an intermediate power flow, the power flow can converge under the same starting conditions. It can obtain a set of convergent power flows that are more similar to the injected data through compressing the error between the intermediate power flow and the initial power flow. When such a power flow is analyzed and compared to the injected data, it is more accurate than the intermediate power flow. In this paper, the form of power flow obtained by compressing the error of the intermediate power flow is called the approximate power flow. Figure 2 shows the interrelationship between injected data, intermediate power flow and approximate power flow.

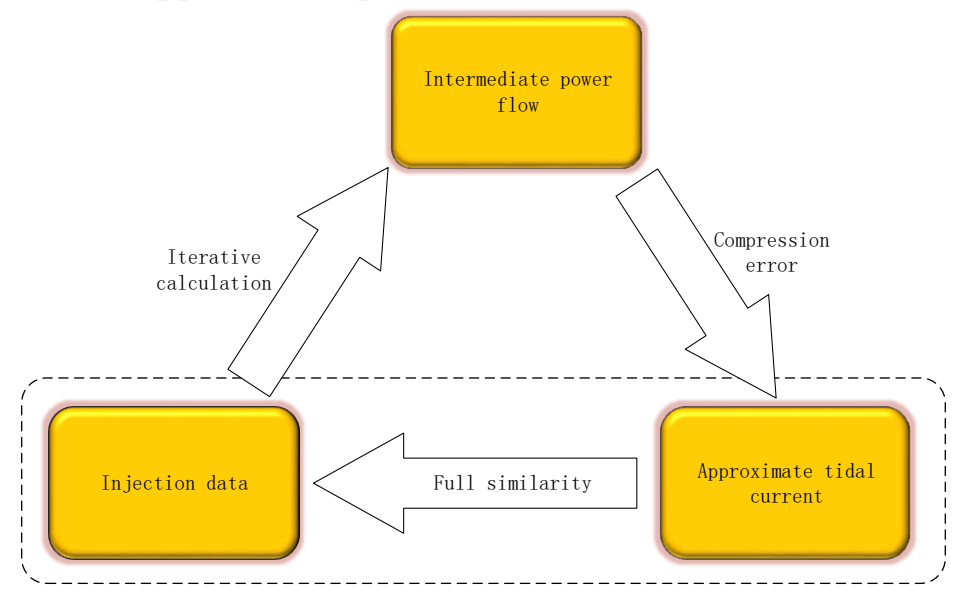

Fig 2: Relationship map

\subsection{Acquisition Method}

Taking the Newton method to calculate the power flow in the polar coordinate system as an example, it is assumed that the initial power flow equation that needs to be adjusted for convergence is:

$$
y^{*}=f\left(x^{*}\right)
$$

After $\mathrm{n}$ iterations, $\mathrm{n}$ linearized approximate intermediate power flows are obtained 


$$
\left\{\begin{array}{c}
y^{1}=f^{1}\left(x^{1}\right) \\
y^{2}=f^{2}\left(x^{2}\right) \\
\cdots \\
y^{n}=f^{n}\left(x^{n}\right)
\end{array}\right.
$$

With the aid of $\Delta y$ reflecting the characteristics of the error relationship between the intermediate flow and the injected data, there are corresponding error evaluation indicators for evaluation:

$$
E_{\max }=\max |\Delta y|
$$

By comparing the minimum index values under each iteration, an intermediate power flow with the smallest error with the injected data can be found, which is described as:

$$
y^{k}=f^{k}\left(x^{k}\right)
$$

The error between the intermediate power flow data and the injected data is $\Delta \mathrm{y}_{\mathrm{k}}$

$$
\Delta y^{k}=y^{*}-y^{k}
$$

The power data of the intermediate power flow is replaced with the original data one by one in the order of $\Delta y$ from large to small, and the power flow calculation is performed until the convergence boundary is reached. The power flow form at this time is defined as the approximate power flow. Compared with the intermediate power flow, the approximated power flow data is closer to the injected data under the premise of ensuring convergence. Therefore, the adjustment action is determined by identifying the system defect of the approximated power flow. Figure 3 below shows the process of obtaining the approximated power flow: 


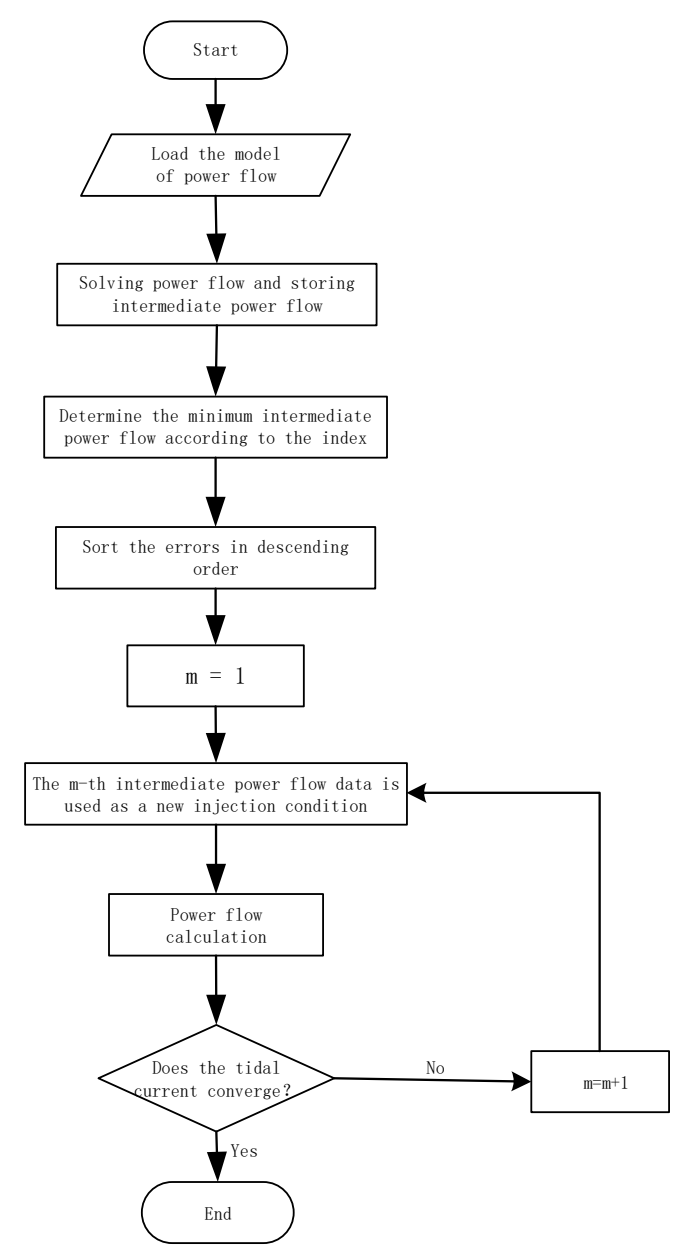

Fig 3: Approximation of the trend of the acquisition flow

\section{RATIONALITY EVALUATION AND ADJUSTMENT ACTION SETTING OF TIDAL CURRENT}

\subsection{Power System Performance Evaluation}

The operating state of the power system is generally divided into five basic operating states: normal state, alert state, emergency state, system crash and recovery state and their relationship is shown in the figure 4 . Whether to meet the corresponding operating constraints is an important evaluation criterion to determine the operating status.

When the system operates stably and economically, the power flow satisfies the constraint conditions of the equation inequality and the system is in a normal state; as the operating conditions gradually deteriorate and the reserve coefficient gradually decreases, it switches to the alert state. In this case, although the constraint conditions are not damage, but the operating economy of the system cannot be guaranteed; when the operating conditions deteriorate further, the inequality constraints will be destroyed, and long-term operation will cause a series of problems such as thermal stability, and the system will enter a state of emergency; if the operating conditions become worse, the system normal operation will not be possible, the equation 
conditions will be destroyed and the system will collapse; if the collapsed power system is restored to normal operation, it needs to go through a recovery state to drive the load again.

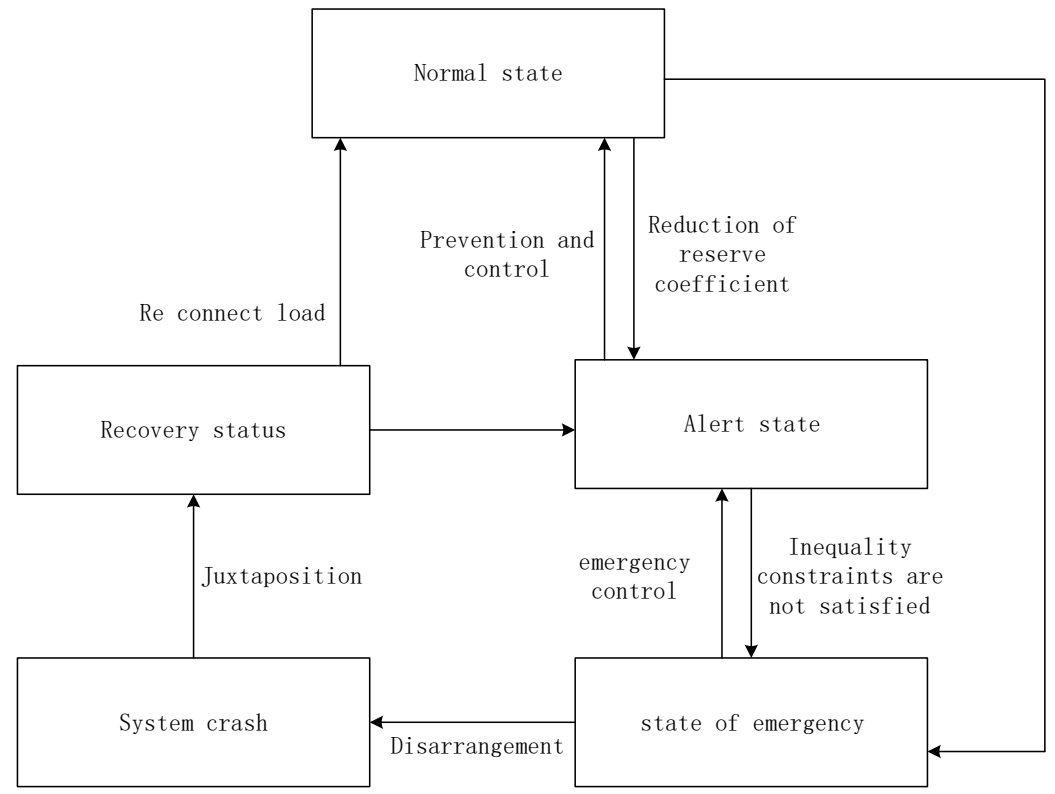

Fig 4: The basic operation of the power system state

The above process can be found that when the normal operation state of the system is gradually broken, the inequality and equality constraints will be gradually broken. At this time, appropriate adjustment actions need to be made to adjust the corresponding abnormal operation state to avoid the grid operation situation.

\subsection{Adjust Action Settings}

\subsubsection{Adjust Generator Output}

In the power flow adjustment, the system power balance can be adjusted by changing the generator power factor. In extreme cases, the generator can also operate as a regulator and only supply reactive power; adjusting the generator excitation current can change the terminal voltage amplitude to improve the reactive power distribution to meet the voltage requirements of the entire system. The adjustment method of adjusting the generator output does not require additional investment and it is flexible and has significant effects which is the first choice for power flow adjustment action selection.

\subsubsection{Regulating Transformer Tap}

As a carrier of energy transmission, the transformer itself does not have the ability to send and receive power but adjusting the transformer tap can improve the regional reactive power distribution and increase the voltage level. Transformer taps should not be adjusted frequently, and improper adjustment may cause voltage instability.

\subsubsection{Compensation Device}

Compensation devices are divided into series compensation and parallel compensation. Among them, series compensation is parameter compensation which is to reduce the reactance and shorten the electrical distance by connecting capacitors in series on the line, but it is generally 
used only as an auxiliary adjustment method because the actual operation is difficult; parallel compensation refers to the use of capacitors and reactors for static reactive power compensators which are connected in parallel in the system to absorb or emit reactive power. Parallel compensation can effectively avoid voltage loss and reduce network losses but its compensation capacity is fixed and increase construction and maintenance costs.

\subsection{Sensitivity Analysis}

The sensitivity matrix is a modification of the Jacobian matrix in the power flow equation. The general formula for power flow calculation is:

$$
\left\{\begin{array}{l}
f(x, u)=0 \\
y=y(x, u)
\end{array}\right.
$$

Where: $\mathrm{x}$ is a state variable, such as node voltage, phase angle, etc.; $\mathrm{u}$ is a control variable, such as generator node active power, voltage, etc.; $y$ is a dependent variable, such as power flowing through a line, etc.

Linearize the power flow equation at the operating point:

$$
\left\{\begin{array}{l}
\Delta x=S_{x u} \Delta u \\
\Delta y=S_{y u} \Delta u
\end{array}\right.
$$

Where $S_{\mathrm{xu}}$ and $\mathrm{S}_{\mathrm{yu}}$ are the sensitivity coefficient matrix:

$$
\begin{aligned}
& S_{x u}=-\left(\frac{\partial f}{\partial x^{T}}\right)^{-1} \frac{\partial f}{\partial u^{T}} \\
& S_{y u}=\frac{\partial y}{\partial u^{T}}+\left(\frac{\partial y}{\partial x^{T}}\right) S_{x u}
\end{aligned}
$$

5.3.1 Load Node Voltage-Generator Terminal Voltage Sensitivity

Reactive power-voltage correction equation:

$$
-L \cdot \Delta V=\Delta Q
$$

There is no generator node in the calculation of the polar coordinate system, so the generator bus is extended to the above formula:

$$
-\left[\begin{array}{ll}
L_{L L} & L_{L G} \\
L_{G L} & L_{G G}
\end{array}\right]\left[\begin{array}{c}
\Delta V_{L} \\
\Delta V_{G}
\end{array}\right]=\left[\begin{array}{c}
\Delta Q_{L} \\
\Delta Q_{G}
\end{array}\right]
$$

When adjusting the generator voltage, assuming that the reactive power of the load bus remains unchanged, there are:

$$
\begin{aligned}
& L_{L L} \Delta V_{L}+L_{L G} \Delta V_{G}=0 \\
& \Delta V_{L}=\left(-L_{L L}^{-1} L_{L G}\right) \Delta V_{G}
\end{aligned}
$$

Let $\mathrm{S}_{\mathrm{LG}}=-\mathrm{L}_{\mathrm{LL}}^{-1} \mathrm{~L}_{\mathrm{LG}}$ and $\mathrm{S}_{\mathrm{LG}}$ be called the generator voltage-load node voltage sensitivity matrix.

5.3.2 Load Node Voltage-Generator Reactive Power Sensitivity 


$$
\begin{aligned}
& {\left[\begin{array}{l}
\Delta V_{L} \\
\Delta V_{G}
\end{array}\right]=-\left[\begin{array}{ll}
L_{L L} & L_{L G} \\
L_{G L} & L_{G G}
\end{array}\right]^{-1}\left[\begin{array}{c}
\Delta Q_{L} \\
\Delta Q_{G}
\end{array}\right]} \\
& =\left[\begin{array}{ll}
R_{L L} & R_{L G} \\
R_{G L} & R_{G G}
\end{array}\right]\left[\begin{array}{c}
\Delta Q_{L} \\
\Delta Q_{G}
\end{array}\right]
\end{aligned}
$$

It is assumed that the reactive power of the load bus does not change, there are:

$$
\left\{\begin{array}{l}
\Delta V_{L}=R_{L G} \Delta Q_{G} \\
\Delta V_{G}=R_{G G} \Delta Q_{G}
\end{array}\right.
$$

Among them:

$$
R_{G G}=\tilde{L}_{G G}^{-1}=L_{G G}-L_{G L} L_{D D}^{-1} L_{L G}
$$

That is equivalent to eliminating the load node and only retaining the equivalent network admittance matrix of the generator node.

5.3.3 Load Node Voltage-Transformer Ratio Sensitivity

Assuming that the micro-increment of the transformer ratio is $\Delta \mathrm{t}$, the generator bus voltage and the reactive power injection amount of the load node do not change at this time the sensitivity relationship can be obtained:

$$
\begin{gathered}
\Delta Q_{L}=\frac{\partial \Delta Q_{L}}{\partial V_{L}^{T}} \Delta V_{L}+\left[\frac{\partial \Delta Q_{L}}{\partial t^{T}}\right] \Delta t=0 \\
T_{L t}=-\left[\frac{\partial \Delta Q_{L}}{\partial V_{L}^{T}}\right]^{-1}\left[\frac{\partial \Delta Q_{L}}{\partial t^{T}}\right]
\end{gathered}
$$

$\mathrm{T}_{\mathrm{Lt}}$ is the sensitivity matrix of load node voltage to transformer ratio.

\section{EXAMPLE ANALYSIS}

The calculation example uses a modified IEEE-39 bus system and the system is shown in Figure 5. The system has ten generators and bus-31 of which is balance bus, the remaining generators are PV bus, and bus- 1 to bus- 29 are PQ buses. The system voltage level is $345 \mathrm{kV}$, and the whole system has 46 lines without transformers. The total load is now doubled, and a normal distribution noise with 0.2 as the mean value and 1 as the variance is applied. The increased power is borne by the generators according to the original power generation ratio. 


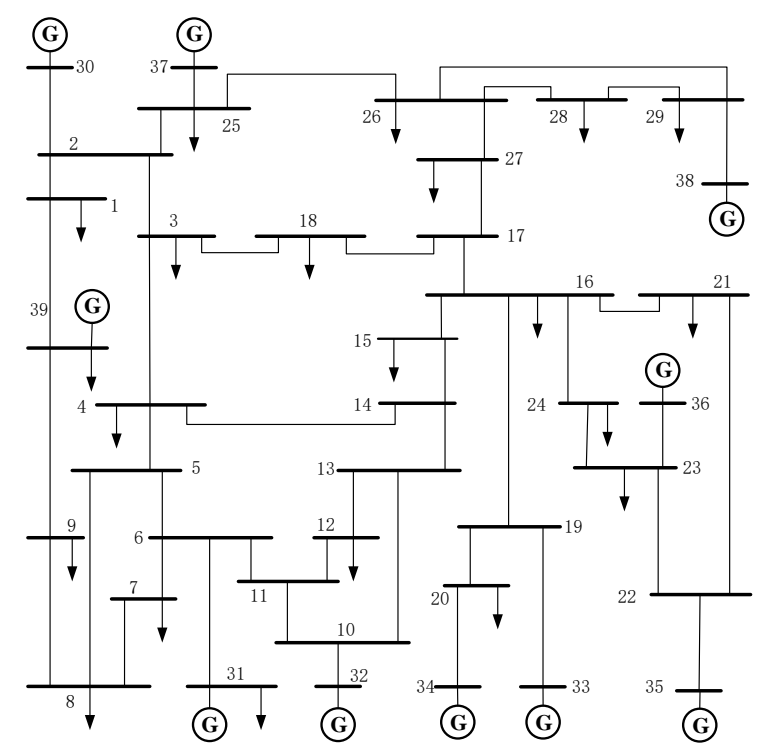

Fig 5: New England 39-bus system

\subsection{Obtaining Approximate Power Flow}

Bringing the above model into the simulation tool for calculation, the power flow has not converged after 20 iterations and the iteration error is shown in Figure 6. According to the indicators mentioned above, the smallest indicator appears in the fifth iteration, and the smallest indicator value is 0.2297 [p.u.].

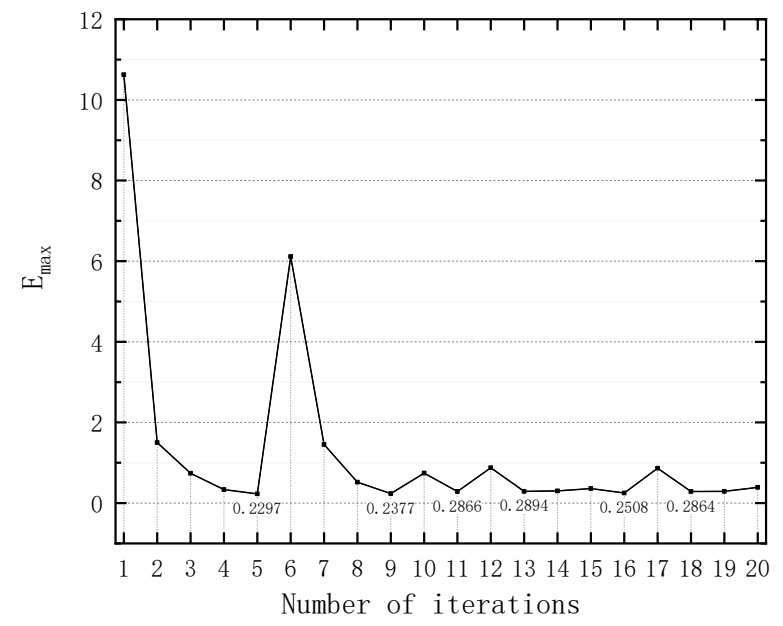

Fig 6: Iterative error curve

According to the approximate power flow acquisition method introduced above, all corrections are sorted from large to small, and the intermediate power flow data is replaced with the injected data according to the sequence, until the convergence boundary is reached, and 38 data replacements are performed when the boundary is reached., The total replacement amount and replacement part are shown in Figure 7. It shows that in this example, after nearly half of the parameters are replaced, a similar power flow is obtained. In other words, in the similar power 
flow, one data that is close to general is the original injection data, which is closer to the injection parameters than the intermediate power flow.

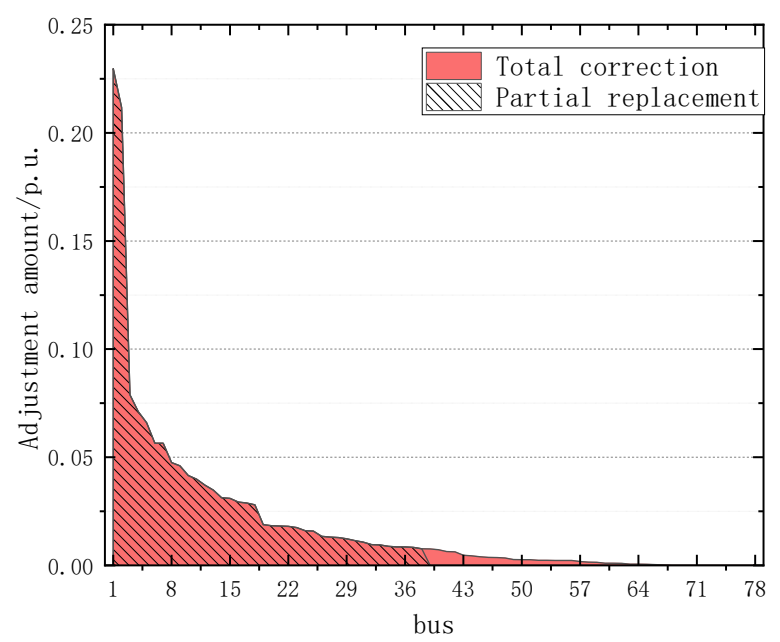

Fig 7: The amount of data replacement

The calculation result of the approximate power flow is shown in Figure 8. From the figure, it can be found that the lowest voltage of the system appears at bus-27 (0.6 p.u.), and the voltage of the bus-26 and bus-17 directly connected to it are seriously low. Therefore, it is judged that the extremely low voltage of node 27 may be the cause of the non-convergence of power flow calculation. Therefore, it is considered to adjust the low voltage of node 27.

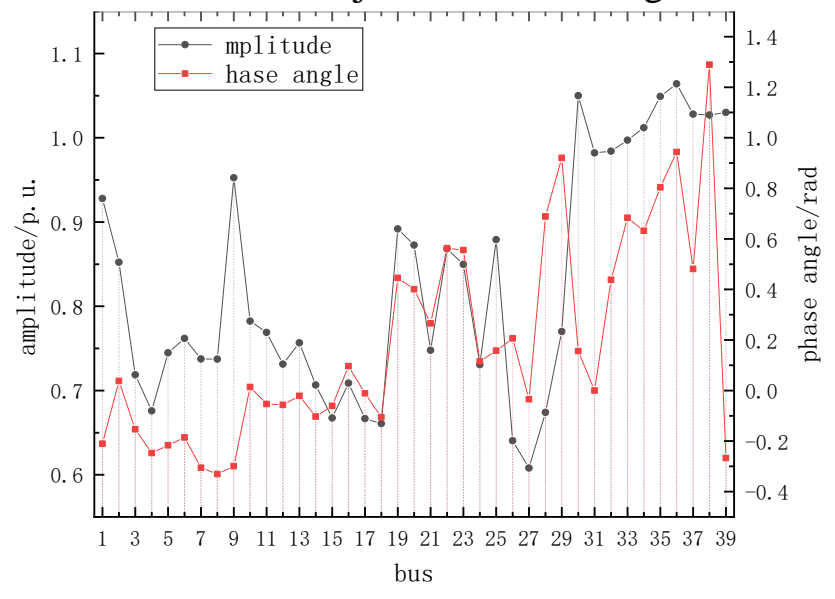

Fig 8: Voltage condition of the acquisition flow

\subsection{Adjust Device Selection}

The method of adjusting the low voltage by changing the voltage of the generator terminal is adopted. The bus-27 and bus-26 with the lowest voltage are selected to calculate the sensitivity of each generator relative to the voltage of the two buses.

To calculate the sensitivity of each generator terminal voltage to the voltage, it is necessary to calculate the augmented matrix as shown in Figure 9. 


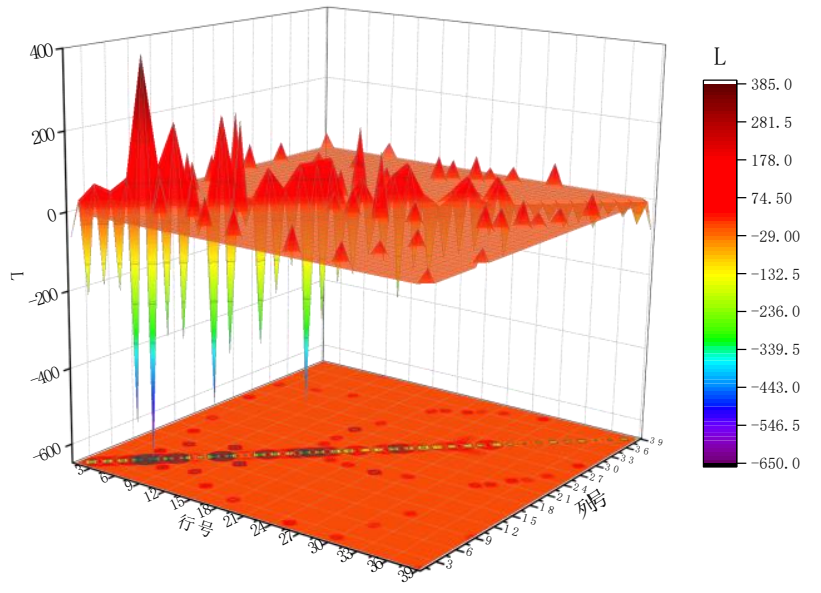

Fig 9: Augmented matrix schematic

According to formula (13), the sensitivity calculation results are shown in Table 1. It can be seen that the voltage of 38-bus has the highest sensitivity to the corresponding load node, so the voltage of 38-bus is selected for adjustment.

TABLE I. No.27 node voltage of the generator terminal voltage sensitivity

\begin{tabular}{|c|c|c|c|}
\hline alternator & sensitivity & alternator & sensitivity \\
\hline Gen30 & 0.150 & Gen35 & 0.120 \\
\hline Gen31 & 0.060 & Gen36 & 0.067 \\
\hline Gen32 & 0.076 & Gen37 & 0.164 \\
\hline Gen33 & 0.114 & Gen38 & 0.255 \\
\hline Gen34 & 0.051 & Gen39 & 0.069 \\
\hline
\end{tabular}

In addition, the 38th generator directly supplies power to the low-voltage area after passing through the bus-29, and the correlation is the strongest, so it is reasonable to judge the required sensitivity.

\subsection{Comparative Display of Adjustment Effect}

Choose to appropriately raise the initial value of PV bus voltage to obtain power flow convergence. In order to modify the original data as little as possible, the power flow is converged when the node voltage setting value is raised by 0.02 [p.u.]. The adjusted system flow is shown in Figure 10. 


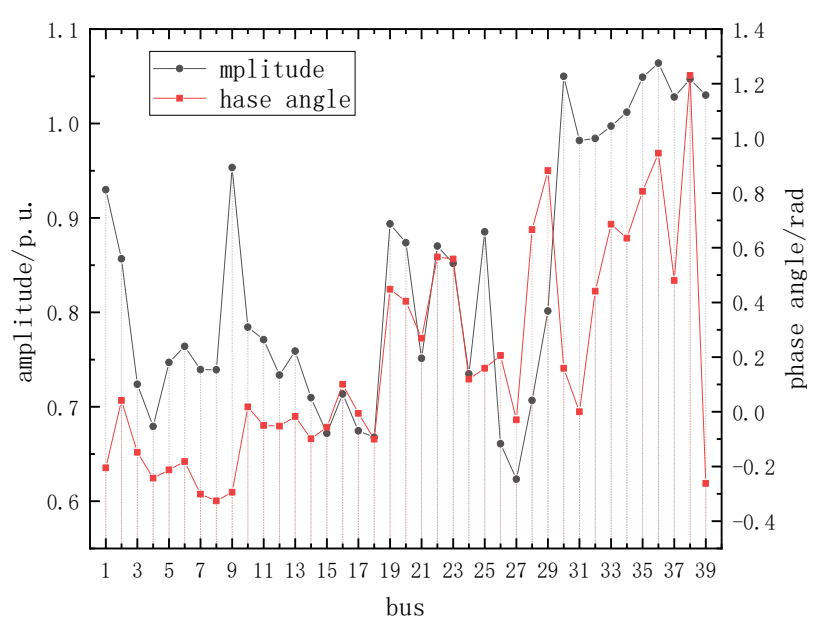

Fig 10: Modified voltage situation

In addition, in order to prove the accuracy of defect recognition and the pertinence of the adjustment strategy, the same adjustment amount is applied to the given voltage of the other eight PV bus. It can be seen from Table 2 that when the same voltage increment ( 0.02 [p.u.]) is applied to other bus, except for the 38 bus, increasing the given voltage of any other bus cannot make the power flow converge.

TABLE II. Adjustment effect of the same adjustment amount applied to different generators

\begin{tabular}{|c|c|c|c|c|c|}
\hline alternator & $E_{\text {Max }}$ & result & alternator & $E_{\text {Max }}$ & result \\
\hline Gen30 & 0.151 & Non convergence & Gen35 & 0.145 & Non convergence \\
\hline Gen31 & - & - & Gen36 & 0.173 & Non convergence \\
\hline Gen32 & 0.158 & Non convergence & Gen37 & 0.146 & Non convergence \\
\hline Gen33 & 0.157 & Non convergence & Gen38 & $2.4 \mathrm{e}-08$ & convergence \\
\hline Gen34 & 0.19 & Non convergence & Gen39 & 0.178 & Non convergence \\
\hline
\end{tabular}

In order to further explore the extent to which the terminal voltage of each generator can be increased to converge the power flow, the traversal method is used to increase the terminal voltage of the generator in steps of 0.01 until the power flow is converged. The terminal voltage adjustment amount of each generator is shown in Figure 11, and mark the two buses voltage sensitivity calculated in Table 2 together in the figure. It can be seen that the bus with higher sensitivity has a smaller adjustment value, while the bus with lower sensitivity has a larger adjustment value. There is a certain degree of negative correlation between terminal voltage adjustment and sensitivity. 


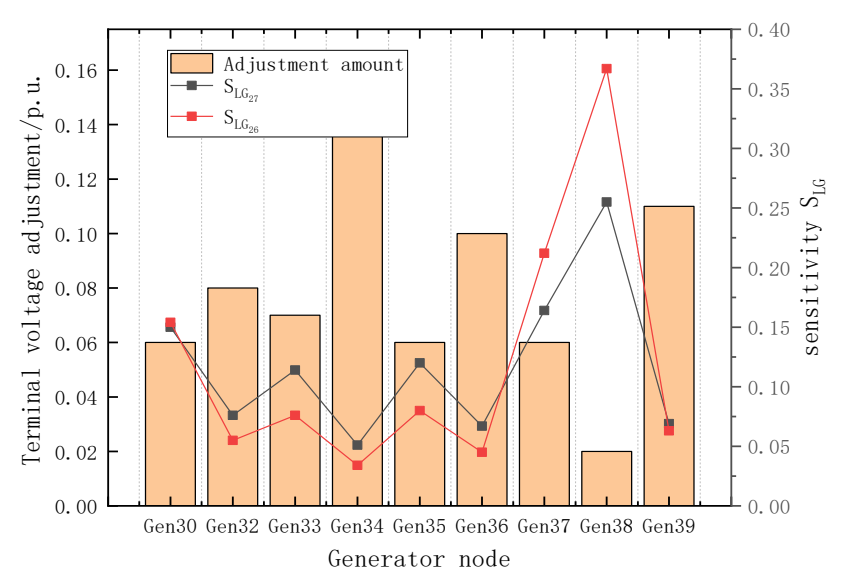

Fig 11: The amount of the sensitivity and the voltage regulator

This shows that the pertinence and accuracy of the adjustment method can be guaranteed.

What needs special explanation is that the analysis method based on the rationality of approximate power flow proposed in this paper only serves the problem of non-convergence adjustment of injected data. The adjustment method focuses on improving the convergence of the power flow. Whether the adjusted system power flow can satisfy the safe and stable operation of the power system is beyond the scope of this article.

\section{CONCLUSION}

This paper proposes the concept of approximate power flow based on the intermediate process of Newton's method. With the help of the voltage problems exposed by the approximate power flow, the voltage sensitivity is used to improve the voltage level of the approximate power flow which indirectly improves the convergence of the power flow and realize the use of electrical knowledge at the physical level to solve the convergence problem at the mathematical level.

The concept of approximate power flow proposed in this paper is completely taken from the intermediate process of power flow iteration and can be automatically realized by programs. It effectively solves the problem of unobservable system state when power flow calculation does not converge, and provides a reference for operators to adjust power flow.

\section{REFERENCES}

[1] Tinney W F, Hart C E (1967) Power Flow Solution by Newton's Method. Power Apparatus \& Systems IEEE Transactions on PAS-86(11): 1449-1460

[2] ZHU Junwei (1995) Power System Analysis. Beijing: China Electric Power Press

[3] Stott B (1971) Effective starting process for Newton-Raphson load flows. Proceedings of the Institution of Electrical Engineers 118(8): 983-987

[4] SUN Qiuye, CHEN Huimin, YANG Jianong, et al. (2014) Analysis on Convergence of Newton-like Power Flow Algorithm. Proceedings of the CSEE 34(13): 2196-2200

[5] Iwamoto S, Tamura Y (1978) A fast load flow method retaining nonlinearity. Power Apparatus \& Systems IEEE Transactions on PAS-97(5): 1586-1599 
[6] Iwamoto S I, Tamura Y (1981) A Load Flow Calculation Method for Ill-Conditioned Power Systems. IEEE Transactions on Power Apparatus and Systems 100(4): 1736-1743

[7] WANG Xianrong, BAO Liming, LIU Zhuo (1994) The study of ill-conditioned load flow using qsasioptimal factor in polar coordinates. Proceedings of the CSEE (01): 40-45

[8] Dehnel M, Dommel H W (1989) A method for identifying weak nodes in nonconvergent load flows. IEEE Transactions on Power Systems 4(2): 801-807

[9] Sasson A M, Trevino C, Aboytes F (1971) Improved Newton's Load Flow Through a Minimization Technique. IEEE Transactions on Power Apparatus and Systems PAS-90(5): 1974-1981

[10] Fletcher R (1971) A General Quadratic Programming Algorithm. Journal of the Institute of Mathematics \& Its Applications 7(1): 76-91

[11] WANG Chengmin, JIANG Chuanwen, HOU Zhijian (2004) The ill-conditioned load flow algorithm based on node lopsided powers. Journal of Shanghai Jiaotong University (08): 1283-1286

[12] Cao J, Yan Z, Fan X, et al. (2015) AC/DC Power Flow Computation Based on Improved LevenbergMarquardt Method. International Journal of Emerging Electric Power Systems 16(1): 1-13

[13] Amini K, Rostami F (2015) A modified two steps Levenberg-Marquardt method for nonlinear equations. Journal of Computational \& Applied Mathematics 288: 341-350

[14] YAN Zheng, FAN Xiang, ZHAO Wenkai (2015) Improving the Convergence of Power Flow Calculation by a Self-adaptive Levenberg-marquardt method. Proceedings of the CSEE 35(08): 19091918

[15] ZHAO Jinquan, CHIANG HsiaoDong, ZHANG Boming (2005) Study on PV-PQ bus type switching logic in power flow computation. Proceedings of the CSEE (1): 54-59

[16] LI Zhihuan, HAN Yunfei, SU Yinsheng, et al. (2015) A Convergence Adjustment Method of Power Flow Based on Node Type Swetching. Automation of Electric Power Systems 39(07): 188-193

[17] Cutsem T V (1995) An Approach to Corrective Control of Voltage Instability Using Simulation and Sensitivity. IEEE Trans on PWRS 10(2): 616-622

[18] Barboza L V, Salgado R (2002) Unsolvable power flow analysis-an approach based on interior pointnonlinear optimization methods Power Tech, IEEE Porto. IEEE

[19] PENG Huimin, LI Feng, YUAN Huling, et al. (2018) Power Flow Calculation and Ill-conditioned Diagnosis for Operation Mode Adjustment of Large-Scale Power Systems. Au-tomation of Electric Power Systems 42(03): 136-142+159

[20] PENG Huimin, YUAN Huling, BAO Yanhong, et al. (2018) Identification and correction method for ill-conditioned power flow of large scale network. Power System Pro-tection and Control 46(22): 116123

[21] AN Jun, SONG Junda, GE Weichun (2020) Convergence identification and adjustment method for power flow calculation in large power grid. Electric Power Automation Equipment 400(2): 103-109 